Pub. Mat. UAB

$N^{\circ} 20$ Set. 1980

Actes VII JMHL

\title{
CLASES DE CHERN DE VARIEDADES PROYECTIVAS
}

Juan A. Navarro Gonzsiez

Dpto. de Algebra y Fundamentos

Universidad de Salamanca

ABSTRACT. Let $X$ be a smooth scheme, projective over a base field, and let $\bar{F}, E$ be locally free $O_{X}$-modules of finite rank. It is shown the rela tion between the graphic of any morphism $F \longrightarrow E$ and the Chern class

$c_{n-r+1}(E-F),(n=\operatorname{rank} E, r=\operatorname{rank} F)$; and, when the morphisa is quite good $c_{n-r+1}(E-F)$ is computed in terms of tinear data on $X$. As a consequence, a method is given to calculate the chern classes of $X$. Finally, it is applied to the Zeuthen-Segre invariant and to Pfaff systems.

Sea $X$ un esquema 1 iso $y$ proyectivo sobre un cuerpo base, $y$.F, $E$ dos ha ces localmente 1 ibres, de $O_{X}$-módulos, de rangos $r$ y $n$ respectivamente. Todo morfismo $\mathrm{f}: \mathrm{F} \longrightarrow \mathrm{E}$ define, de modo natural, una grafica proyectiva en $\mathbb{P}(F \oplus E)$; la clase de cohomología de dicha grắica, en el graduado de la teoria $k$, se denota $F_{f}$.

$\Gamma_{f}$ es un elemento de $G K(\mathbb{P}(F \oplus E))$ homogéneo de grado $n ; y$ se verifica, siendo $\pi: \mathbb{P}(F \oplus E) \rightarrow X$ la proyección natural:

Teorena: en $G K(X): \pi_{\pi}\left(\Gamma_{F} \cdot \Gamma_{0}\right)=c_{n-r+1}(E-F)$.

La demostración está basada en el teorema de periodicidad, que detemina la estructura del graduado de la teoría $K$ de un fibrado proyectivo, y en la consideración de la zona del infinito de $\mathbb{P}(F \oplus E)$.

Cuando $\mathrm{F}$ es inyectivo, $0 \longrightarrow \mathrm{F} \stackrel{\mathrm{f}}{\longrightarrow} \mathrm{E} \longrightarrow \mathrm{E} / \mathrm{F} \longrightarrow 0$, 1a clase de Chern $c_{n-r+1}(E / F)$ se puede calcular mediante haces coherentes, bajo ciertas condiciones (más débiles que imponer la transversalidad de $f$ respecto del norfismo nulo):

Teorena: Si el haz $\operatorname{Ext}^{3}\left(\mathrm{E} / \mathrm{F}, \mathrm{O}_{X}\right)$ está concentrado en codimensión $n-r+1$, y es 1-generado en los puntos genëricos de su soporte, se verifica: 


$$
c_{n-r+1}(E / F)=\sum_{p} \ell(\underbrace{\operatorname{Ex} E^{1}}\left(E / F, O_{X}\right)) \cdot p
$$

Ia sunu extendida a los puntos genéricos del soporte de $\operatorname{Ext}^{1}\left(\mathrm{E} / \mathrm{F}, \mathrm{O}_{\mathrm{X}}\right)$. Este teorema es consecuencia del anterior, porque, con las condiciones inpuestas, la gráfica proyectiva de $f$ y del morfismo nulo se cortan sin tores. En consecuencia $\Gamma_{\mathrm{f}} \cdot \Gamma_{0}$ es fácil de calcular en teoría $K$. La condición de que $\operatorname{Ext}^{\prime}\left(E / F, O_{X}\right)$ sea l-generado, en los puntos genéricos de sa soporte, es necesaria para que el corte de las gráficas proyectivas mencionadas sea de igual dimensión que su proyección por $\pi ; y$, en consecuencia, poder calcular con facilidad $\pi_{\star}\left(\Gamma_{\mathrm{f}} \cdot \Gamma_{0}\right)$.

La aplicación más importante de este mêtodo es el câlculo de las clases de Chern de cualquier variedad proyectiva $X$ :

Proyectando desde una cadena de subvariedades lineales del espacio ambiente, se obtienen aplicaciones racionales $x \rightarrow \mathbb{P}_{r}, 1 \leqslant r \leqslant \operatorname{din} x=n$. $\mathrm{Si} \mathrm{U}_{r}$ es el abierto mäs grande en que está definida la proyección racional $X \longrightarrow \mathbb{P}_{r}$, se imponen las condiciones:

a) $\mathrm{U}_{\mathbf{T}} \longrightarrow \mathbb{P}_{\boldsymbol{Y}}$ es un morfismo denso y separable.

b) La ramificación de $U_{r} \longrightarrow \mathbb{P}_{\tau}$ tiene codimensión $n-r+1 ; y$ la variedad inneal que se uciliza para proyectar sobre $\mathbb{E}_{r}$, no corca a ia ramisicaciön de $u_{r+1} \longrightarrow \mathbb{P}_{t+1}$.

c) $\operatorname{Ext}^{\prime}\left(\Omega_{U_{r}}^{1} / \mathbb{P}_{r}, O_{U_{r}}\right)$ es 1 -generado en los puntos genéricos de su soporte.

Con las condiciones impuestas, si $\delta_{n-r+1}$ es la clase de equivalencia racio nal del ciclo definido por $\operatorname{Ext}^{1}\left(\Omega_{U_{r}}^{1} / \mathbb{P}_{r}, O_{U_{r}}\right)$, se verifica:

$$
c_{n-r+1}\left(\Omega_{X}^{1}\right)=\delta_{n-r+1}-\sum_{i=1}^{n-r+1}\left(\begin{array}{l}
r \\
i
\end{array}\right)\left(\frac{1}{i-n}\right)^{i} c_{n-r+1-i}\left(\Omega_{X}^{1}\right)
$$

siendo $H$ una sección hiperplana de $X$. En particular, si para cada número natural $m, z_{\text {th }}$ es un subesquema cerrado $y$ liso de $x$, contenido en $Z_{m-1}$ y cuya clase de equivalencia racional es $H^{\mathrm{n}}$, se verifica:

$$
c_{n-r+1}(x)=\left[\sum_{i=1}^{n+r+1}(-1)^{i+1}\left(\begin{array}{l}
r \\
\sum_{n-r+i-i}
\end{array} c_{n}\left(2_{i}\right)\right]+(-1)^{n-r+1} \delta_{n-r+1}\right.
$$

Estas fórmulas permiten computar las clases de Chern de $x$ en función de los ciclos de ramificación de las proyecciones racionales $x \rightarrow \mathbb{P}_{Y}$, de 1 a sección hiperplana $\mathrm{H}$, $\mathrm{y}$ de sus intersecciones. 
Definiciones: $S i$ es un divisor de $x$, se define $c_{k}(D)$ como el elemen ro de grado $k+1$ de $G K(X)$ definido por:

$$
c_{k}(D)=(-1)^{k} \sum_{j=0}^{k}(-1)^{j} c_{j}(x) \cdot D^{k+1-j}
$$

cuando $D$ es una hipersuperficie lisa de $X$, coinciden con las clases de Chern de $D$, consideradas en $X$.

$\mathrm{Si} \omega$ es una 1 -forma sobre $\mathrm{X}$, su haz de 1innea asociado $(\omega)$ es:

$$
(\omega)(U)=\left\{f \in \Sigma(U):\left\{\cdot \omega \varepsilon \Omega_{X}^{\prime}(U)\right\}\right.
$$

siendo $\Sigma$ el haz de funciones racionales sobre $X$. El divisor asociado a (w) se llama divisor de $w$.

$\mathrm{Si} \omega$ es una 1-forma sobre $\mathrm{X}$ con singularidades aisladas, $\mathrm{y} D$ es su divisor, se verifica:

$$
c_{n}(X)=(-1)^{n}\left(\sum_{p \in X} n_{p} \cdot p\right)+c_{n-1}(-D)
$$

siendo $n_{p}$ la longitud de $\operatorname{Ext}^{2}\left(\left(\Omega_{X}^{2} /(\omega)\right)_{p}, 0_{p}\right)$ sobre $0_{p}$. Este numero $n_{p}$, cuando $w$ es una diferencial exacta: $w=d f$, coincide con el número de Milnor de $f$ en $p$. En particular, para diferenciales exactas sobre superficies, se obtiene el cläsico invariante de Zeuthen-Segre:

Si f es una función racional sobre la superficie $x$, cuyas fibras son re ducidas, y tal que el divisor de polos es una curva $\gamma$, de gênero $g$ :

$$
g I c_{2}(x)=\sum_{p} n_{p}-2 \gamma^{2}+4 g-4
$$

Los números de Milnor $n_{p}$ se pueden calcular facilmente en este caso:

a) Si $\mathrm{p}$ es un punto singular de una curva de nivel de $f$, $\mathrm{n}_{\mathrm{p}}=\ell\left(0_{\mathrm{p}} /\left(\frac{\partial f}{\partial \mathrm{x}}, \frac{\partial f}{\partial y}\right)\right)$, siendo $\mathrm{x}, \mathrm{y}$ coordenadas locales en $\mathrm{p}$. Este número puede calcularse siempre mediante la teoría italiana de intersección de curvas planas, utilizando transformaciones cuadrâticas ([1]) . b) Si $p$ es un punto base de $f, y f=g / h$ siendo $g, h$ funciones regulares en $p$ sin ceros comunes, se verifica:

$$
\mathrm{n}_{\mathrm{p}}=\ell\left(0_{\mathrm{p}} /\left(\mathrm{h} \frac{\partial \mathrm{g}}{\partial \mathrm{x}}-\mathrm{g} \frac{\partial \mathrm{h}}{\partial \mathrm{x}}, \mathrm{h} \frac{\partial \mathrm{g}}{\partial \mathrm{y}}-\mathrm{g} \frac{\partial \mathrm{h}}{\partial \mathrm{y}}\right)\right)
$$

y este número se puede calcular, a su vez, por transformaciones cuadräticas. 
Volviendo al caso general (dim $X=n$ ) , cuando se consideran 1-formas exactas, se obtiene:

$\mathrm{Si} f$ es una función racional, sus fibras son reducidas, las singularidades de df son aisladas, y su divisor de ceros $D$ es simple y corta transver salmente al de polos, se verifica:

$$
c_{n}(X)=(-1)^{n}\left(\sum_{p \varepsilon X} n_{p} \cdot p\right)+2 c_{n-1}(D)-c_{n-2}(D \cap D)
$$

siendo $n_{p}$ el número asociado a df.

Todas las demostraciones de los enunciados anteriores se obtienen al considerar la sucesión exacta:

$$
0 \longrightarrow(\omega) \longrightarrow \Omega_{x} \longrightarrow \Omega_{x}^{\prime} /(w) \longrightarrow 0
$$

$y$ determinat $c_{n}(\Omega /(\omega))$ mediante datos lineales sobre $x$, taI $y$ como hemos mostrado que se puede hacer.

\section{REFERENCIAS:}

[1] F. Enriques - Teonía geometrica delle equazioni e delle funzioni algebriche. Ed. Aicola Zanichelli. Bologna, 1918.

[2] J. Milnor - Singular points of complex hipersurfaces. Annais of Math. Studies, 61, Princeton University Press, 1968.

[3] R. Thon - Les ensembles singuliers d'une application ... Séminaire de Topologie de Strasbourg, Dec. 1957.

[4] I.R. Porteous - Todd's canonical classes. Proceedings of Liverpol singularities. L.N.M., $n^{\circ}$ 192, Springer-Verlag. Berlín. 